\title{
A Robotic Arm Based Design Method for Modular Building in Cold Region
}

\author{
Zexin Sun ${ }^{1,2,3}$, Hongyuan Mei ${ }^{1,2,3}$, Wente Pan ${ }^{1,2,3, *}$, Zhengwei Zhang ${ }^{1,2}$ and Jie Shan ${ }^{1,2}$ \\ 1 School of Architecture, Harbin Institute of Technology, Harbin 150001, China; dyzmother@sina.com (Z.S.); \\ meihongyuanadri@126.com (H.M.); archzkk@163.com (Z.Z.); 17b334002@stu.hit.edu.cn (J.S.) \\ 2 Key Laboratory of Cold Region, Urban and Rural Human Settlement Environment Science and Technology, \\ Ministry of Industry and Information Technology, Harbin 150001, China \\ 3 Architectural Design and Research Institute of HIT, Harbin 150090, China \\ * Correspondence: panwente@gmail.com; Tel.: +86-13936333319
}

check for updates

Citation: Sun, Z.; Mei, H.; Pan, W.; Zhang, Z.; Shan, J. A Robotic Arm Based Design Method for Modular Building in Cold Region.

Sustainability 2022, 14, 1452. https:// doi.org/10.3390/su14031452

Academic Editors:

Siu-Kit (Eddie) Lau, Vesna Kosorić, Abel Tablada, Zdravko Trivic,

Miljana Horvat, Milena Vukmirović, Silvia Domingo-Irigoyen, Marija Todorović, Jérôme H. Kaempf, Kosa Golić and Ana Peric

Received: 2 December 2021

Accepted: 21 January 2022

Published: 27 January 2022

Publisher's Note: MDPI stays neutral with regard to jurisdictional claims in published maps and institutional affiliations.

Copyright: (c) 2022 by the authors. Licensee MDPI, Basel, Switzerland. This article is an open access article distributed under the terms and conditions of the Creative Commons Attribution (CC BY) license (https:// creativecommons.org/licenses/by/ $4.0 /)$.

\begin{abstract}
The robotic arm has emerged as an essential tool for the rapid construction of high-quality buildings due to its ability to repeat instructions, achieve precise positioning and fine operations. The robotic arm can also effectively replace workers to complete building construction under low temperature and short daylight conditions. Thus, it can be forward-looking to construct modular buildings in cold region, but how to realize the construction of modular buildings through humanmachine coordination and remote operation is a significant issue. This article discusses the feasibility of robotic arm assembly design methods in the field of architecture by simulating the complete design and construction process of modular buildings. According to parameterized module design, a three-dimensional computer model is transformed into an electronic file that directs the action of the robotic arm to complete assembly via a custom processing program. This article details a theoretical and practical exploration of the construction of modular manipulators and has certain guiding significance for the intelligent design and construction of future buildings.
\end{abstract}

Keywords: construction in cold region; modular building design; robotic arm

\section{Introduction}

As a high-precision, multi-input, and multi-output, highly nonlinear, and strongly coupled complex system, the robotic arm is widely used in industrial assembly, hazardous environments, and other fields due to its unique operational flexibility. The progress of technology has prompted people to have higher requirements for their living environment, not only at the aesthetic level, but also in terms of functionality, requiring buildings to provide a safer, more comfortable, and more convenient environment. At the same time, the increasingly fast pace of life has catalyzed the efficiency of building construction. Therefore, higher requirements for buildings, such as standardized design, refined construction, and rapid construction have emerged as mainstream construction trends in recent years. The development of a standard construction process from computer digital modular design to robotic arm construction can facilitate the application and promotion of the robotic arm in the construction field to meet these demands.

\subsection{Modular Design}

The concept of modular design originates from industrial design and refers to the deconstruction of the overall product into a series of modular units based on different functional analyses within a certain range. Different design can then be formed through the selection and combination of modules [1]. Modular design is widely used in electrical appliances, machinery, aviation, and other fields, and is conducive to the serialization, combination, generalization, and standardization of industrial production [2]. Since Ford adopted the modular design method to build the production line in 1913, architects have 
attempted to employ modular design to realize the standardization and economy of buildings [3]. The modular construction of buildings is relevant in a large range of scenarios. It involves the combination of independent standardized units with similar sizes and properties through the design of a complete system to form a whole building [4]. Compared with traditional building design, modular design sometimes has the following advantages: (1) Economic advantages: building modules can be prefabricated in a factory, and the assembly line method greatly reduces building materials and construction time; (2) reproducibility: as modular design adopts a universal structure, the building modules can be replaced and recycled, and the building can also be reorganized; (3) ecological advantages: modular buildings use emergent design ideas, the buildings are variable, and the building area can be continuously adjusted according to the actual needs of the building's functions. The traditional modular building construction process is based on the functional requirements proposed by the client in which the architect designs the modules, the factory prefabricated unit processing, and the construction party performs on site assembly. This form of multiparty cooperation creates a unified modular building pattern. An architectural plan that is too standardized will fail to adapt to the individualized and differentiated needs of modern society $[5,6]$. Therefore, a transformation from mechanical production to digital manufacturing is underway to meet these demands.

\subsection{Robotic Arm}

The application of automation and robotics in the manufacturing industry is profoundly affecting the development of architectural design. Robotic arms are the hallmarks of general-purpose machines. They can accept instructions to accurately locate a point in three-dimensional (or two-dimensional) space for operations and integrate advanced control technology and a memory system that can accurately complete complex motion instructions many times. As a combination of automation and robots, robotic arm construction technology can effectively liberate building design from the constraints of traditional architectural methods as an emerging technology in the field of building design [7]. The robotic arm can realize operations similar to that performed by a human arm and can replace humans in situations requiring heavy labor [8]. Compared with traditional manufacturing, robotic arms have the advantage of completing tasks more quickly and working uninterrupted $24 \mathrm{~h}$ a day. The robotic arm has higher accuracy, which can ensure that the structure and shape of the product are consistent in quality. It can also replace humans in complex and dangerous environments.

Connecting robotic arms with modular building design can provide revolutionary breakthroughs. A number of explorations and applications of robotic arms in this field have already occurred. In 1985, Warzawski pioneered the application of robotic arms in the field of construction engineering, discussing its advantages in high-quality construction, labor-saving, and risk reduction [9]. In the past 40 years of subsequent development, many excellent cases of robotic arm intervention in building design and construction have been presented. Dutch designer Joris Laarman used a robotic arm to build a 12-m long steel bridge, realizing the construction of complex shapes [10]. The team at Tongji University in China used human-machine collaboration to fabricate a bridge and realized the construction of a larger span [11]. A joint experimental project between America's building design company SOM and Princeton University with Delft University in the Netherlands used a robotic arm to complete three-dimensional cast glass masonry [12]. The 3D printing company ICON and BIG building design company cooperated with NASA to design a lunar architectural plan based on 3D printing [13].

In theatrical aspect, Cambridge handbook in construction robotics book series provides a comprehensive, systematic and detailed discussion of the application of robots in engineering. Among them, robot-oriented design is more for theoretical perspective, which discusses the mechanized ideas in the processes of creativity, design, construction, and management [14]. The construction Robots discusses the types of construction robots and the combination of site construction from the perspective of specific applications [15]. 
A research team from Inha University explored the feasibility of robotic arms for building construction by conducting several experimental tests on robotic arms that perform building installation [16]. The University of Alberta scholars have also proposed a more efficient solution to the collision problem that robotic arms may face when building prefabricated modular components [17].

\subsection{Advantages of Robotic Arms in Building Design and Construction}

The robotic arm is highly applicable to modular design, which requires a high level of precision. The standardized preparation of modules and precise connecting of components can be completed using a manufactural robotic arm in a factory. This process is the foundation of the overall construction of a building. The robotic arm can also realize the high-precision and rapid assembly of modules. In this process, operating the robotic arm through the numerical control program can more accurately locate building modules and achieve precise equipment selection. Thus, combining a robotic arm with modular design can help achieve high-precision, low-cost, and rapid building construction [18].

In addition to realizing free form and precision machining, the robotic arm also has the capacity to innovate the conceptual design process. Architects can consider the construction process at the beginning of design and write computer "programs" to directly command the robotic arm to participate in design and construction. In this process, the theory, design, materials, manufacturing, and construction are considered together as a whole. As a result, mass production is naturally transformed into mass customization [19]. Thus, modern industrial machinery technology could realize the digital processing and manufacturing of nonstandard modules through control coding. Compared with the traditional modular building design and construction methods, the combination of robotic arms and modular building has the advantage of higher flexibility, which will improve the diversity of results [20].

The advantages of robotic arms in modular buildings located in cold region are mainly reflected in the following three aspects. First, the robotic arm can carry out construction in extreme environments and complex conditions, such as replacing workers in extremely cold or dark areas. Second, the robotic arm has higher construction accuracy. Compared with humans, the robotic arm can perform high-precision and repetitive tasks with minimal errors. Third, the robotic arm can carry out more complex construction, and combined with a generation program, it can realize the construction of complex shapes in a threedimensional space.

This research aims to provide a complete design method for the robotic arm to intervene in the design of modular buildings in cold region to realize unmanned construction of modular buildings. Previous research into the subdivision of modular buildings using robotic arms has been carried out by university architectural research institutes, such as The Bartlett Faculty of the Built Environment BC4 research course at University College London. However, existing research results have largely focused on the masonry process of small bricks or blocks [21]. There remains a lack of research on the mechanical installation of the overall module. Thus, this research focuses on using the robotic arm to assemble the overall module and integrating the robotic arm concept into the entire building design process. In addition, as extreme weather in cold region presents higher requirements for the design and construction of manipulators, construction in cold region is another key aspect of this work.

\section{Methodology}

\subsection{Work Flow}

Before proposing a modular cold region building design method using robotic arms, it is necessary to analyze the similarities and differences between cold region construction and non-cold region construction. The construction of robotic arms in cold region mostly employs the remote operation mode. Thus, the program must be set in advance so that the robotic arm can complete the construction autonomously. Therefore, in addition to the 
construction function, the robotic arm should also have error monitoring and self-correction capabilities. In the construction environment, the construction manipulator should have the ability to build at ultra-low temperatures, and its electrical components should have a certain degree of self-deicing ability. In terms of the combination of modules, as most buildings in cold region have a centralized layout, multiple module groups should be closely integrated, and internal modules must be installed first. Therefore, the overall construction route needs to be reasonably designed in advance.

Based on the high correlation between modular design and robotic arm construction and its role in the design and construction of cold region, the design of modular cold region using robotic arms should adopt the following workflow.

1. Deconstruct the function of the building, establish the construction environment and the scale of the building through a systematic analysis of the overall building task, and dismantle the overall task.

2. Classify the functional space, disassemble the task functions of the building into different types of building modules, and configure the corresponding traffic and service modules.

3. Reconstruct the organizational order, set the parameters of the different types of building modules to be formed, determine the association and separation relationship, and set the proportion of the module in the overall building.

4. Generate the construction program, determine the construction space domain through the construction environment in the first step, and reduce the negative space to form an overall frame. Generate a continuous space sequence under the overall frame and reasonably fill the space sequence with modules.

5. Simulate module assembly by determining the storage space of the construction materials (modules) and the operating space of the robot arm. Employ this as a basis to combine the multi-manipulator coordination technology and error monitoring technology to realize the simulation assembly (Figure 1).

\subsection{Man-Machine Collaboration Method}

The six-dimensional robotic arm can be used to perform the construction tasks for the building boxes. To complete the tasks, the robotic arm must move in a predefined trajectory that avoids possible collisions with the other boxes and equipment. As shown in Figure 2, the red dashed line represents the desired trajectory with the start point $\left(x_{0}, y_{0}, z_{0}\right)$ and the end point $\left(x_{f}, y_{f}, z_{f}\right)$. The construction problem is then reduced to a control design that drives the robotic arm to follow the desired trajectory. To this end, the dynamics of the robotic arm described by a differential equation and a classic controller can be easily designed. First, the dynamic model of the robotic arm is given according to the following equation [22]:

$$
M(q) \ddot{q}+C(q, \dot{q}) \dot{q}+g(q)=\tau-\tau_{b}
$$

where $M(q)$ is the inertia matrix, $C(q, \dot{q}) \dot{q}$ is the vector of centrifugal and Coriolis forces, $g(q)$ is the gravitational forces, $\tau$ is the control torque, and $\tau_{b}$ is the external torque imposed by the boxes. Let $s_{d}$ represents the desired trajectory, which can be inversed to the desired joint movement by making use of the following relationship:

$$
q_{d}=\varphi\left(s_{d}\right), s_{d}(0)=\left(x_{0}, y_{0}, z_{0}\right), \quad s_{d}(t)=\left(x_{f}, y_{f}, z_{f}\right)
$$

where the function $\varphi$ represents the inverse kinematics of the robotic arm which can be determined based on the geometrical parameters and structure of the robotic arms. The joint position error

$$
\widetilde{q}=q_{d}-q
$$


which is measurable and employed to design the controller based on classic PID control and the advanced adaptive control methods. PID control refers to a control system that controls the proportion, integral and derivative of the error generated by comparing the information collected in real-time data of the controlled object with the given value in the industrial process control. PID control has the advantages of simple principle, strong robustness, and wide range of practicality. In the following, the PID-based control method is given according to the research results in [23]:

$$
\tau=M\left(q_{d}-\widetilde{q}\right) K_{1} \widetilde{q}+M\left(q_{d}-\widetilde{q}\right) K_{2} \dot{\widetilde{q}}+M(q) \ddot{q}_{d}+C(q, \dot{q}) \dot{q}+g(q)+\tau_{b}
$$

where $K_{1}$ and $K_{2}$ are the tuning control parameters, the tracking position error $\widetilde{q}, \ddot{q}_{d}, \tau_{b}$ can be measured or computed via sensors. With the control design, the robotic arm can finish the building construction with satisfactory performance, theoretically guaranteeing complex automatic construction in the cold region.

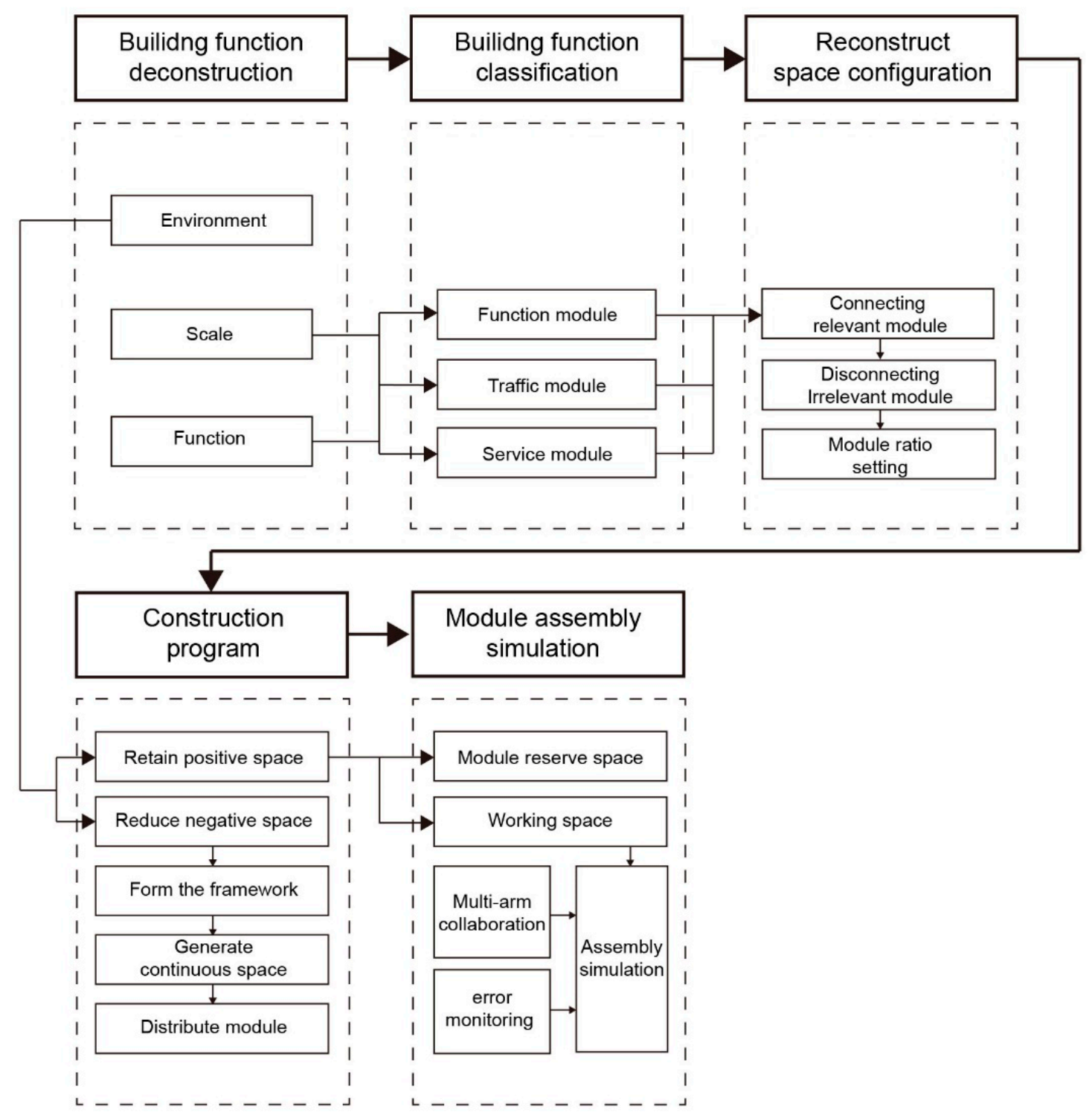

Figure 1. The workflow of modular building design. 


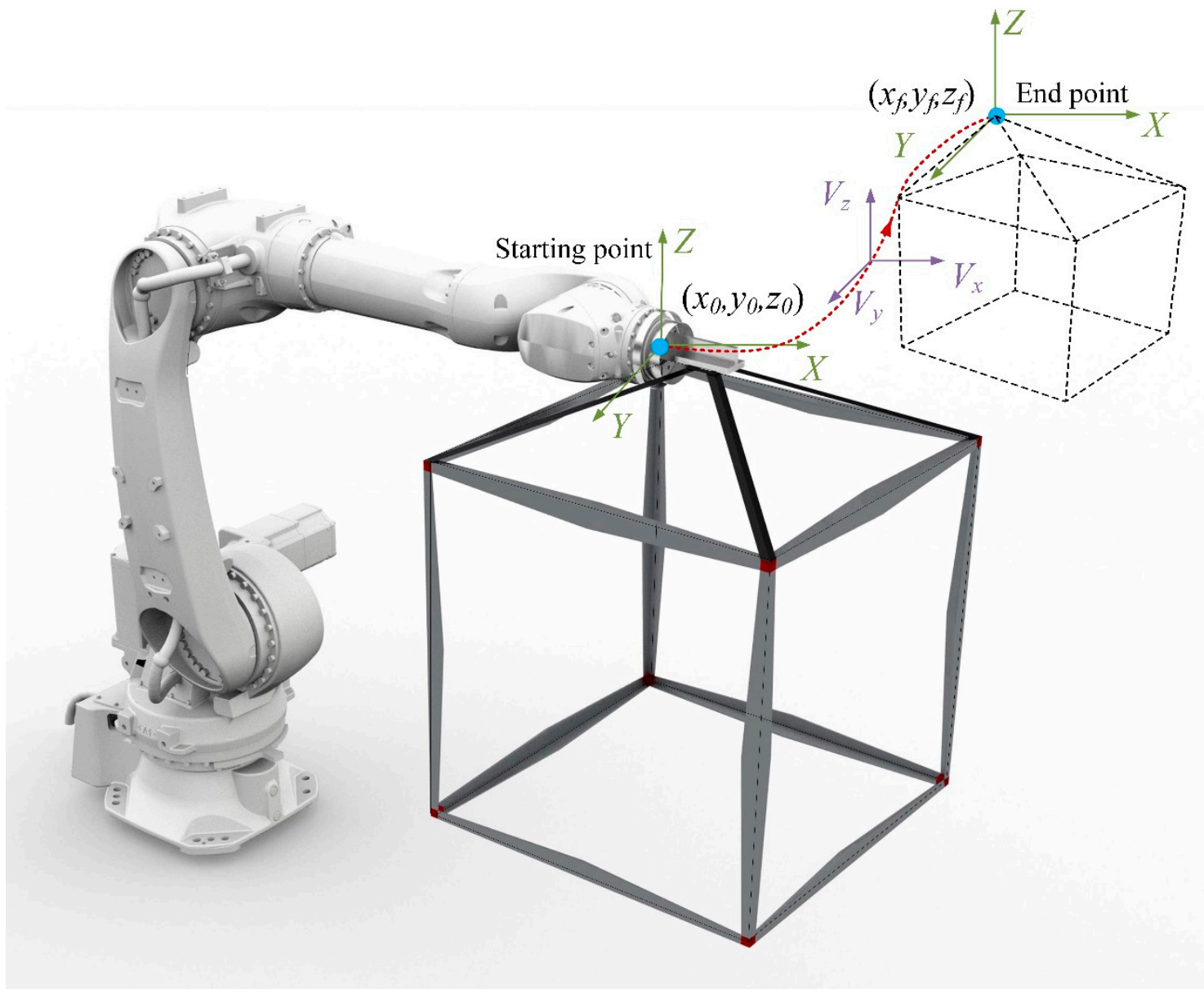

Figure 2. Robotic operation route.

\subsection{Case Study}

The exhibition area of a typical cold museum building is used as the research case in this article. The site is selected for two primary reasons. First, there are different types of functional spaces in museum buildings, which can be summarized into four categories: exhibition space, service space, horizontal transportation, and vertical transportation. Fewer space types are the basis of modular building design. Second, according to different building types, buildings can be divided into strong control buildings and weak control buildings. Strong control buildings mean that the spatial function layout and flow organization of the building are greatly affected by the building type, and the building must comply with strict specifications. Thus, the design randomness is weak, such as in schools and hospitals. Conversely, weakly controlled buildings are those where the spatial layout and crowd organization are less affected by the type of building, and the design of the building has a certain degree of autonomy and randomness. Compared with strong control buildings, museum buildings are weak control buildings that provide a certain degree of autonomous operation for the reorganization of modules.

This article uses the location of Harbin, a cold region city in the northeast of China, as an example in our deconstruction of modular buildings that contain complex spaces. The building is broken down into modules with different functions and recombined after optimization. We explore a set of "codes" that conform to morphological generation and structural logic and use Grasshopper as the operating platform to direct the robotic arm to simulate assembly. 


\section{Robotic Arm Assembly Simulation Experiment}

\subsection{Modular Building Deconstruction and Classification}

Modularity is the operational logic of top-down decomposition of a system layer by layer to solve complex problems, which aims to decompose complex system processing into more manageable modules. Drawing on this logic, the museum is first decomposed into $3 \mathrm{~m} \times 3 \mathrm{~m} \times 3 \mathrm{~m}$ conceptual modules based on its size, and then the decomposed modules are classified based on the different functions of the museum: functional space module, public space module, horizontal traffic module, vertical traffic module, etc. In order to make the deconstructed space more adaptable to the needs of modular architecture, we use the polyominoes and polycubes mathematical models [24] to make integrated functional modules with generalized, standardized, and adaptable properties for robotic arm construction. Different modules can be freely combined and expanded to produce a variety of spatial possibilities. In this scheme, the building modules can adopt a steel structure frame, using 10-mm thick variable cross-section L-shaped steel, and the main materials can be connected by cube members with a side length of $100 \mathrm{~mm}$, and a cube module with a side length of $3 \mathrm{~m}$ can be formed finally, details shown in Figure 3 .

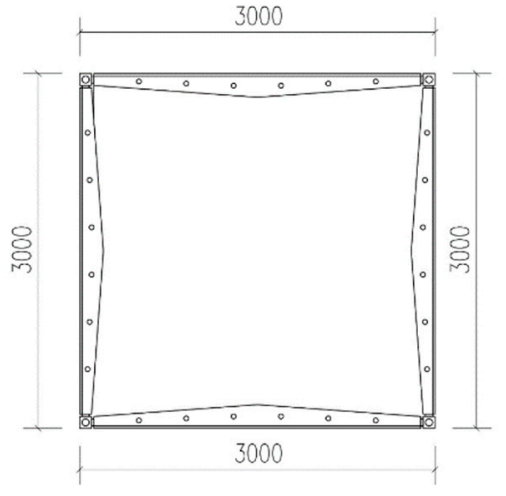

(a)

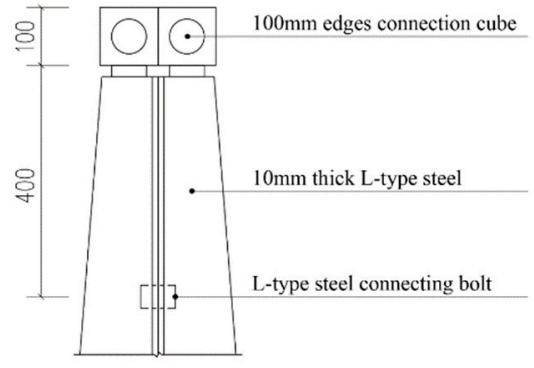

(b)

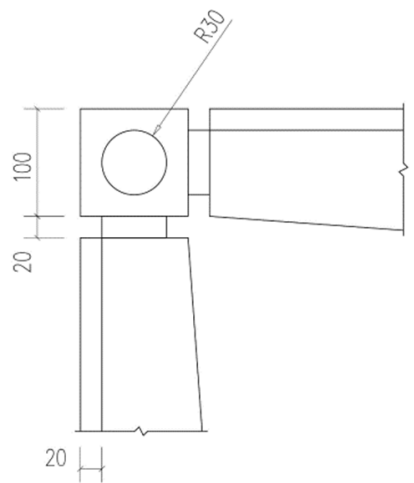

(c)

Figure 3. Module detail. (a) Module layout. (b) Components. (c) Components connection mode.

The horizontal traffic module and functional space module are defined as families A and $B$, which can be arranged at ground level or $1 / 2$ level locations. The vertical traffic module is defined as family $C$, which connects the ground level and the $1 / 2$ level by stairs or elevators. The remaining modular spaces, i.e., public space modules, are defined as family D. Finally, the museum is simulated, and the modules of each family are connected to each other. The polyominoes and polycubes methods are used in the mathematical model [24] to make the integrated functional modules appear generalized and standardized. Adapt to the needs of robotic arm construction. Different modules can be freely combined and grown, creating a variety of space possibilities. These modules are only a simple simulation of the basic space of the building. However, the simulation results in this paper can be seen as an abstract "code" logic, and the realization of the real building will require further refinement and order reconstruction.

\subsection{Modular Building Order Reconstruction}

Combining the module parameters together is vital to further explore the logic of the "code" generation. We introduce a mathematical permutation algorithm based on the logic of combining multiple cubes in three-dimensional space to form an unknown architectural space. The process uses two generators in the VBScript, the Line and Point (L.P.) Generator and the Family Generator. 
The design process is as follows. An L.P. Generator is used to generate random lines in a specific domain, and a cube is obtained based on the control points. The cube is replaced with modules, and the paths are interpolated and considered as combinations of different families. Using the family calculator, a combination of modules is selected, and the building is generated. Under this reconstruction logic, modules are free to grow, while realistic conditions and subjective ideas act as the only controlling factors. The method of generating modular buildings is deduced based on this logic. The complete space is formed as a solid by the division of the modular combinations, and the part that is divided out can be seen as a negative space, which is considered an outdoor area. The modules that combine the paths become the positive space of the traffic area if they are present in the indoor circulation space of the building, and the intermediate space between the positive and negative space can be used as a functional space. The robotic operation route is shown in Figure 4.

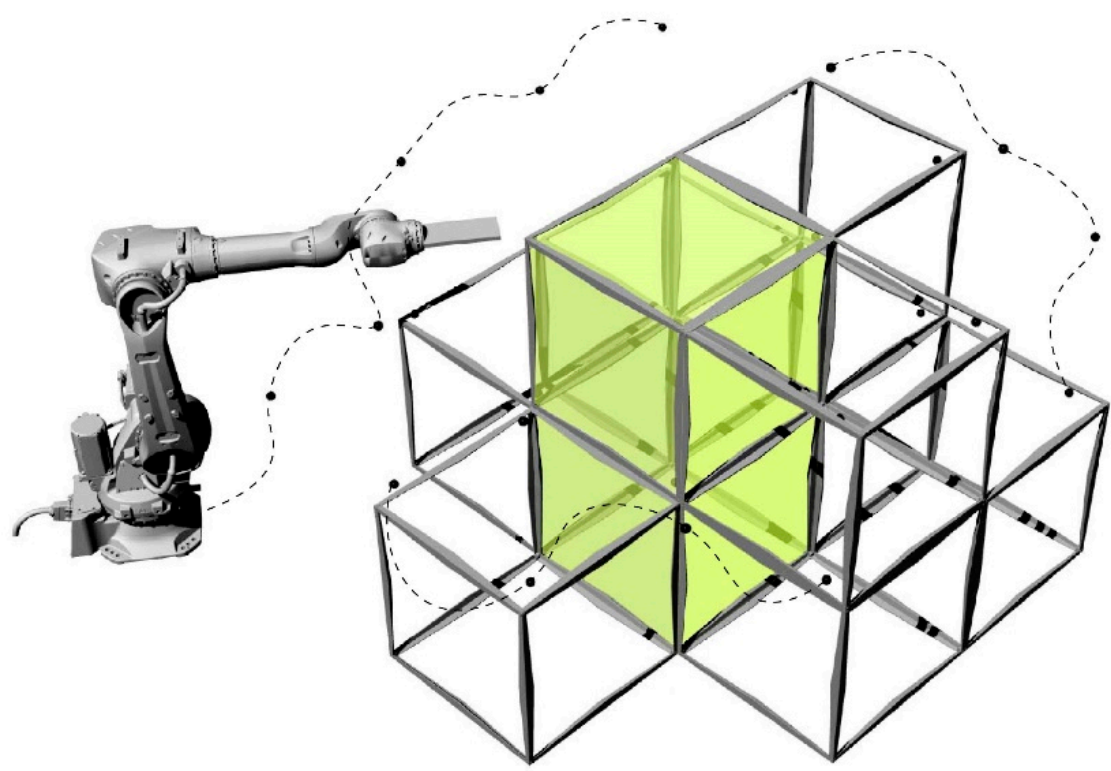

Figure 4. The module construction process.

On the basis of the previous steps, the four families could be then developed into more precise modules to simulate actual buildings. Some parts are the connection space, where new families E, F, G are created as other traffic connection module. A total of eight groups and 20 modules are included in the system. Combining parameter modules is a key step to further explore the "code" generation logic. Here, we introduce the mathematics permutation and combination algorithm, based on multicube combination logic in the three-dimensional space to form an unknown architectural space. In this process, two calculators (generator) are used in the VBScript, the dot-line calculator (L.P. Generator) and the family calculator (Family Generator). Design process: Use L.P. Generator to generate random lines in a specific field, and get cubes based on control points. Replace the cubes with modules, intersperse paths, and treat them as a combination of different families. Use the family calculator to select a combination of modules to generate a building. As far as the first logic is concerned, modules grow freely, and realistic conditions and subjective ideas are the only controlling factors. Based on this logic, the generation method of modular building is deduced. The complete space is divided into cubes through the division of obstacles, and the divided part can be regarded as a negative space and as an outdoor space. If the module that combines the path presents the building's indoor circulation space, it becomes a positive space and serves as a traffic space. The intermediary space between the positive space and the negative space can be used as a functional space. 


\subsection{Module Growth "Code" Design}

The process of module "generating" can be summarized as follows:

1. Determine a reasonable spatial domain as the usable space of the building;

2. Subtract the negative outdoor space to get the indoor space;

3. Use the previous generator to design a reasonable continuous space as circulation space so that the remaining area can be used as functional space;

4. The positive circulation space, the middle functional space, and the negative outdoor space will constitute the whole building (Figure 5).

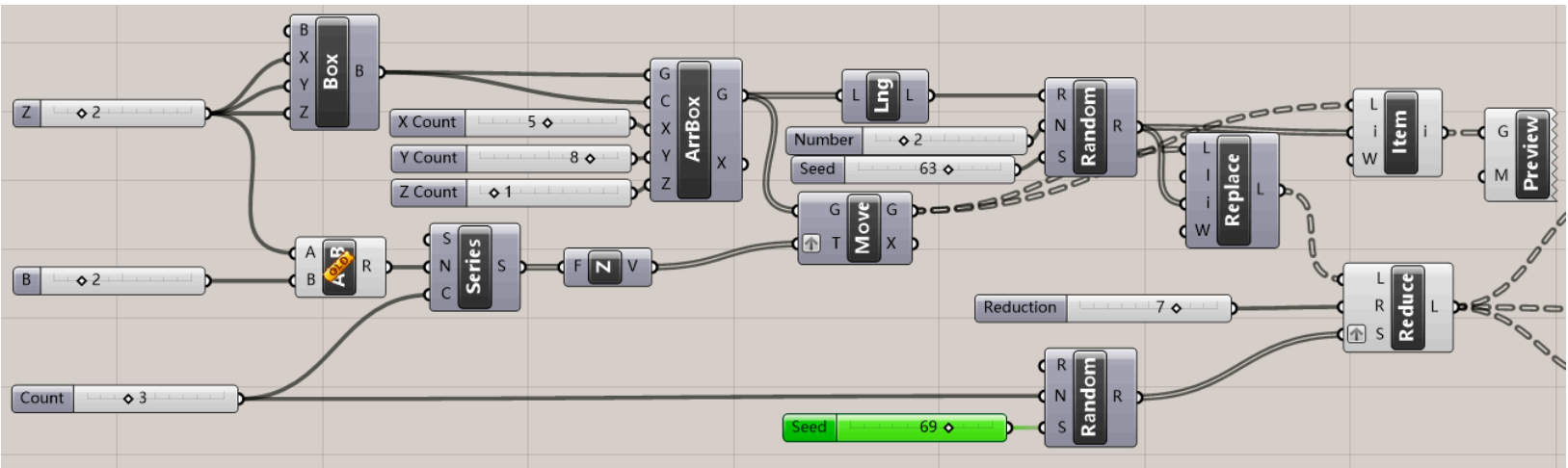

(a)

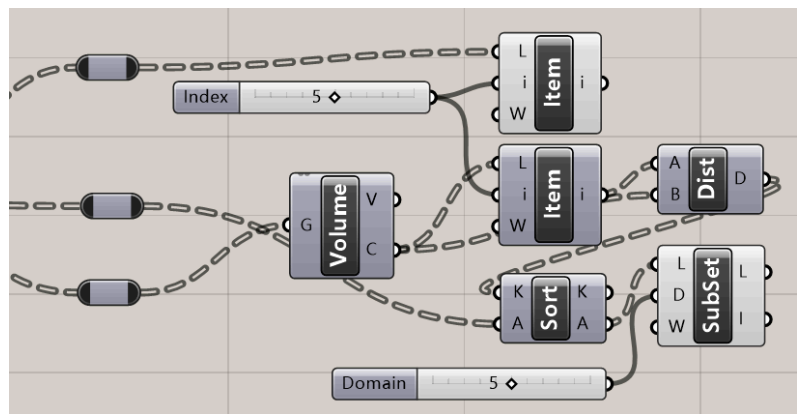

(b)

Figure 5. Module growth code by grasshopper. (a) Constructive part; (b) Generative part.

This design approach increases the freedom and diversity of modular building results. Among them, the generated modular building may produce many results because the shape and position of the module combination can be easily controlled, and the continuous space is randomly generated. The results can be optimized to enhance variation of the original single path forming a linear space, where the original modules can be replaced with a third interface to enrich the spatial variation by using specific polycubes (tetra-cubes). For modular buildings containing complex flow lines, in cases where multiple paths exist in the building, they can be calculated separately and combined and connected with X-modules as joint spaces at the intersections. The $\mathrm{R}$ module may be any of the previous race modules, and attention should be paid to its continuity in multiple directions. Thus, the "code" design of the modular building is completed.

\subsection{Assembly Simulation of Modular Buildings}

In this section, we simulate the assembly of a small museum. The entire building is in a cube of $15 \mathrm{~m} \times 24 \mathrm{~m} \times 9 \mathrm{~m}$. The external space is removed to obtain the internal space, and then the appropriate continuous space is calculated as circulation space, and the rest is used as functional space. The functional space can be used as video rooms, storage, staff rooms, toilets, etc. The circulation space is the main exhibition space, which contains six different flow lines that can be combined to form a 3D exhibition traffic network. Figures 6-8 show 
that the relationship between the various streamlines is close, and the spatial streamlines are rich and diverse.

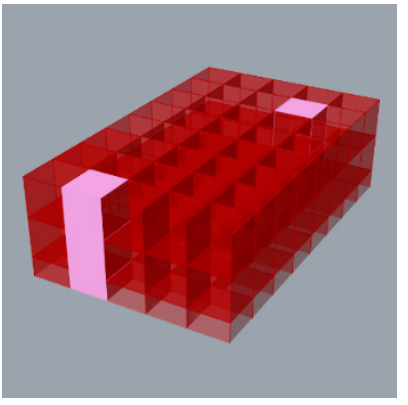

(a)

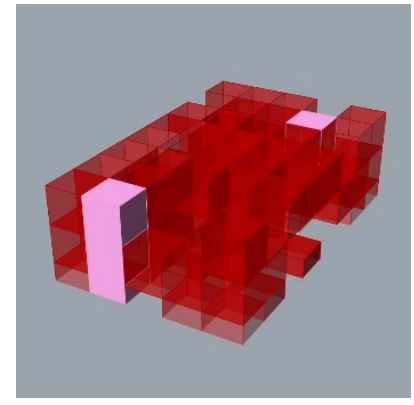

(b)

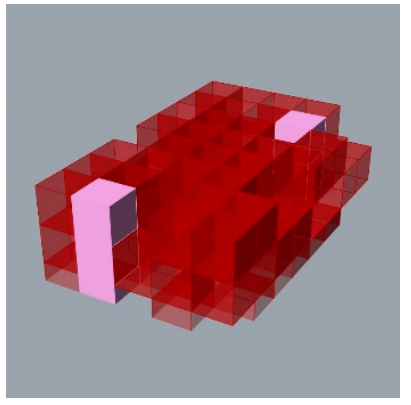

(c)

Figure 6. Generated building frame type A. (a) Unabridged; (b) reduce 10; (c) reduce 15.

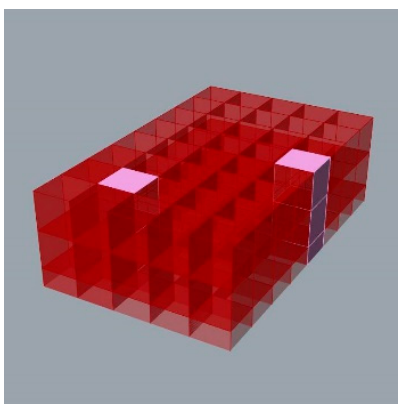

(a)

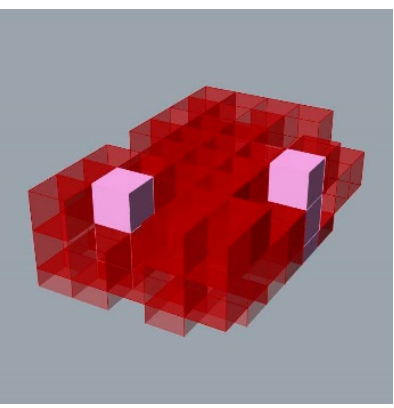

(b)

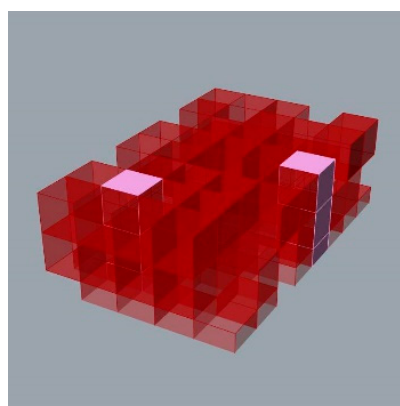

(c)

Figure 7. Generated building frame type B. (a) Unabridged; (b) reduce 10; (c) reduce 15.

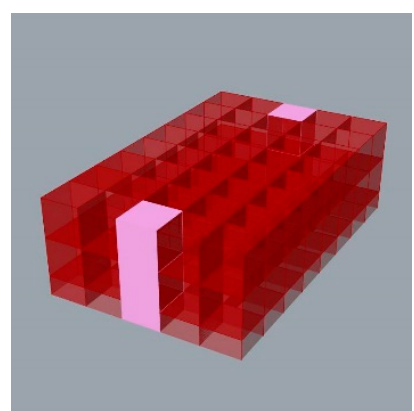

(a)

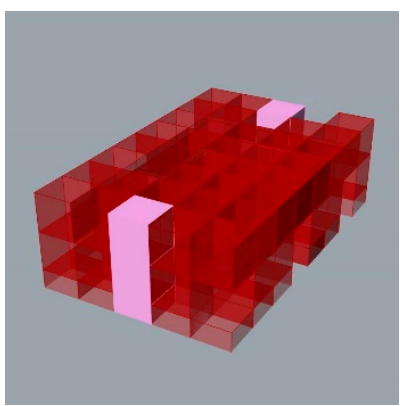

(b)

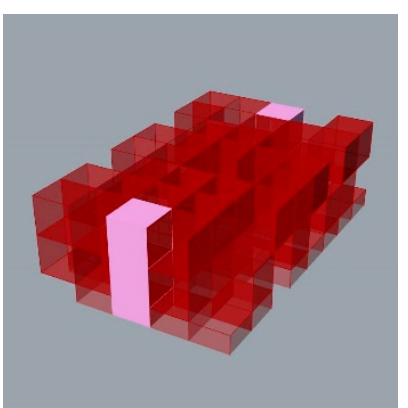

(c)

Figure 8. Generated building frame type C. (a) Unabridged; (b) reduce 10; (c) reduce 15.

\subsection{Modular Construction and Assembly Using Robotic Arms}

In the robotic arm assembly simulation experiment, the module is designed to be realistic and simple to operate, and the construction process is as close to reality as possible. The module is of medium size, with a side length of $50 \mathrm{~mm}$. Three-dimensional printed sticks (linkage rods) and nodes are used as the structure of the module, both with magnets inside to increase the stability of the structure. Steel balls with a diameter of $10 \mathrm{~mm}$ are placed at the apex of the module as joints to connect the different modules. Interior floors, stairs, and walls are simulated with laser cut wooden panels. The ABB Robotics IRB 120 robotic arm, combined with the tool, is employed for the assembly. The tool magnetically attracts the joints of the cube using four steel screws and connects the steel balls using a large magnet with the middle screw. 
As the computer randomly generates all positions of the different modules, a strategy for assembly needs to be developed for the path design of the robotic arm. First, a set including all six families and steel balls is designed. The specific operational paths are then designed with a computer using Grasshopper to simulate the generation of the module building by "code". The numbers of all families of modules are translated into a process code that directs the movement of the robot arm to automatically calculate the route of all robotic arms.

Finally, the museum exhibition hall in Harbin is simulated by connecting modules of different "clans" together (Figure 9). These modules only perform a simple simulation of the basic space. Therefore, the simulation result in this article is only regarded as an abstract "code" standard, and the realization of the real architecture will require further refinement.

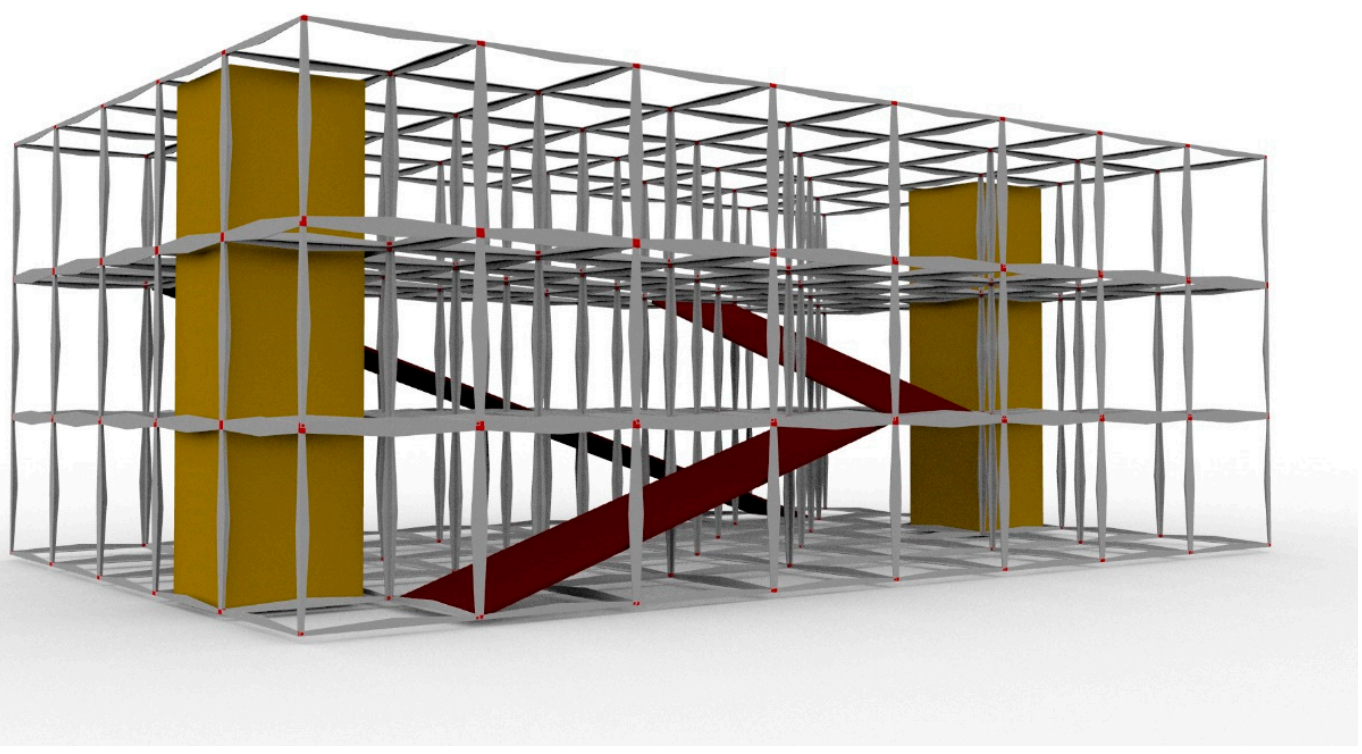

Figure 9. One design proposal based on simulation result.

\section{Discussion and Conclusions}

The method described in this work provides a new way for manipulators to intervene in architectural design. It is a way to achieve a direct link between computer design models and modular construction. Thus, it requires the adoption of new design strategies and techniques.

The recommended design strategies for the design of modular buildings using robotic arms are summarized as follows. First, a prefabrication strategy should be adopted. The core modules of the building should be prefabricated in a factory to minimize operations on the construction site. Building modules should have a high degree of integration, including structures, maintenance walls, various equipment, etc., and should also be prefabricated in the modules in advance. Second, a standardized strategy should be adopted. Building modules with uniform standards can be replaced between different buildings, can be reused many times, and have material-saving efficiency. Third, sophisticated strategies should be adopted for building modules in all aspects of their design, production, and assembly. This will result in reduced errors in the module components so that they can be accurately assembled into a whole onsite.

The following design methods are recommended for modular building design using robotic arms. First, the method of man-machine coordination should be employed so that construction staff can control robotic arms in real-time and make quick adjustments to the preset programs according to the onsite situation. The mechanical arm should also have a certain protection function. Thus, when other people or equipment enter its operating range, it will automatically stop. Second, the module emergence method could be used. By combining the preset parameters, the intelligent emergence of modular combination methods through the program will form a variety of conceptual design schemes. The 
architect can then compare and optimize according to the generated schemes. Third, intelligent supervision methods can be used. Camera equipment can be set on the robotic arm and combined with cloud computing for automatic image recognition to check the construction process and avoid incorrect construction in real-time.

At present, the application of robotic arms to modular construction still has certain limitations. While manipulators have been widely used to replace human manual operations in the industrial production of automobiles and household appliances, their application in building construction faces continued challenges. As buildings are unlike industrial products and often unique in design, the robotic arm operating system must be repeatedly redesigned. In this case, the load of the robotic arm needs to reach $827 \mathrm{~kg}$ to carry the weight of a modular steel frame, and the span of the robotic arm needs to reach $8 \mathrm{~m}$ to realize the hoisting of the modular building. There are still challenges in using a huge robotic arm to achieve construction in cold region, and key technologies still need breakthroughs. Additionally, as the building module itself has a relatively large mass and volume, the assembly of the building module by a robotic arm requires a high load capacity. Finally, the precision operation requirements of construction have not yet been met. However, as the technology progresses, robotic arms will increasingly participate in construction to meet demands for high-quality environments, the industrial transformation of buildings, and construction technology development.

This article systematically discussed the development processes of modular building and robotic arm intervention in architectural design research and listed out the advantages of combining the two. A man-machine collaboration method using robotic arms to design modular buildings in cold region was put forward. Employing the case study of a museum in Harbin, the design and construction process was completely simulated, and the design inspiration and application limitations were discussed. This research provided a preliminary exploration of the involvement of robotic arms in the design of modular buildings in cold region to inform the design and construction of buildings through mechanical engineering experience.

Author Contributions: Conceptualization, Z.S., H.M. and W.P.; methodology, Z.S., H.M. and W.P.; software, Z.S. and W.P.; validation, W.P., Z.Z. and J.S.; formal analysis, J.S.; investigation, W.P. and Z.Z.; resources, Z.S. and W.P.; data curation, W.P.; writing-original draft preparation, Z.S. and W.P.; writing-review and editing, W.P.; visualization, Z.Z.; supervision, H.M.; project administration, H.M.; funding acquisition, H.M. All authors have read and agreed to the published version of the manuscript.

Funding: This research was funded by [National Natural Science Foundation of China] grant number [52008128].

Institutional Review Board Statement: Not applicable.

Informed Consent Statement: Not applicable.

Data Availability Statement: Not applicable.

Conflicts of Interest: The authors declare no conflict of interest.

\section{References}

1. Kawecki, L.R. Environmental Performance of Modular Fabrication: Calculating the Carbon Footprint of Energy Used in the Construction of a Modular Home. Ph.D. Thesis, Arizona State University, Phoenix, AZ, USA, 2010.

2. Ahn, Y.H.; Kim, K. Sustainability in modular design and construction: A case study of the stack. Int. J. Sustain. Build. Technol. Urban Dev. 2014, 5, 250-259. [CrossRef]

3. Molavi, J.; Barral, D.L. A Construction procurement method to achieve sustainability in modular construction. Proc. Eng. 2016, 145, 1362-1369. [CrossRef]

4. Generalova, E.M.; Generalov, V.P.; Kuznetsova, A.A. Modular buildings in modern construction. Proc. Eng. 2016, 153, 167-172. [CrossRef]

5. Cameron, P.J.; di Carlo, N.G. Piecing Together Modular: Understanding the Benefits and Limitations of Modular Construction Methods for Multifamily Development. Master's Thesis, Massachusetts Institute of Technology, Cambridge, MA, USA, 2007. 
6. Yuan, F.; Meng, Y. The methodology of digital modular fabrication based on the BIM platform. Time Archit. 2013, 3, 30-37. (In Chinese)

7. Gambao, E.; Balaguer, C.; Gebhart, F. Robot assembly system for computerintegrated construction. Autom. Construct. 2000, 9 , 479-487. [CrossRef]

8. Chen, H. Research on realization of manipulator and its control system. J. Hubei Corresp. Univ. 2015, 1, 172-173. (In Chinese)

9. Warszawski, A. Economic implications of robotics in building. Build. Environ. 1985, 20, 73-81. [CrossRef]

10. Wolzak, T. Robots Complete Span of 3D-Printed Bridge for Amsterdam Canal. Dezeen. Available online: https://www.dezeen. com/2018/04/17/mx3d-3d-printed-bridge-joris-laarman-arup-amsterdam-netherlands (accessed on 10 November 2021).

11. Tian, F. Two Robotic Fabrication Methods Entwine to Make Tongji University Bridge. Dezeen. Available online: https://www. dezeen.com/2019/11/29/ robotic-fabricated-hybrid-bridge-technology (accessed on 10 November 2021).

12. Oikonomopoulou, F.; Bristogianni, T. Glass Vault. Parametric House. Available online: https:/ / parametrichouse.com/glassvault/ (accessed on 10 November 2021).

13. ICON Team. ICON Receives Funding from NASA and Launches "PROJECT OLYMPUS" to Reach for the Stars with Off-World Construction System for the Moon [EB/OL]. Available online: https://www.iconbuild.com/updates/icon-receives-fundingfrom-nasa-and-launches-project-olympus (accessed on 21 May 2021).

14. Bock, T.; Linner, T. Robot-Oriented Design: Design and Management Tools for the Deployment of Automation and Robotics in Construction Cambridge University Press: Cambridge, UK, 2015.

15. Bock, T.; Linner, T. Construction Robots: Volume 3: Elementary Technologies and Single-Task Construction Robots, 1st ed.; Cambridge University Press: Cambridge, UK, 2016.

16. Park, S.; Park, J.H.; Jung, S.; Ji, S.J. Foam cutting for an architectural installation using industrial robot arm: Calibration, error, and deviation analysis. Autom. Constr. 2022, 133, 103986. [CrossRef]

17. Yang, C.H.; Kang, S.C. Collision avoidance method for robotic modular home prefabrication. Autom. Constr. 2021, 130, 103853. [CrossRef]

18. Terada, Y.; Murata, S. Automatic modular assembly system and its distributed control. Int. J. Robot Res. 2008, 27, 445-462. [CrossRef]

19. Sun, C. When architects start coding an introduction to Gramazio \& Kohler's practices. Time Archit. 2012, 9, 46-49. (In Chinese)

20. Yin, H.; Qu, M.; Zhang, H.; Lim, Y. 3D Printing and Buildings: A Technology Review and Future Outlook. Technol. Archit. Des. 2018, 2, 94-111. [CrossRef]

21. Claypool, M.; Retsin, G.; Garcia, M.J.; Jaschke, C.; Saey, K. Automation and the Discrete: Exploring New Potentials for Streamlining Production in Architectural Design Research. J. Archit. Educ. 2021, 75, 108-114. [CrossRef]

22. Murray, R.M.; Li, Z.; Sastry, S.S. A Mathematical Introduction to Robotic Manipulation; CRC Press: Boca Raton, FL, USA, 1994.

23. Kelly, R.; Santibanez, V.; Loria, A. Control of Robot Manipulators in Joint Space; Springer: London, UK, 2005.

24. Fan, Z. Polycube Parameterization Automatic Construction and Cross Parameterization. Master's Thesis, Zhejiang University, Hangzhou, China, 2006; pp. 19-23. 